\title{
Analysing phenotypic variation in Eucalyptus pauciflora across an elevation gradient in the Australian Alps
}

\author{
Giles Young, Islay Andrew, Kristi Lee, Xiaoyn Li, Rachael Robb, \\ Isabella Robinson, Holly Sargent, Bronte Sinclair
}

\section{Abstract}

Research related to the phenotypic plasticity of species that face rapidly changing conditions in the near future, such as those in the Australian Alps, is extremely important for ecological conservation efforts. Eucalyptus pauciflora is a species found throughout much of the Australian Alps. In this paper, the phenotypic plasticity exhibited by the species in terms of tree height and leaves across an elevation gradient was studied to gain insight into how the species is able to survive in such a range of conditions. Height and leaf size decreased with elevation, chlorophyll content (a measure of photosynthetic potential) increased, while specific leaf area (indicative of investment in photosynthesis and growth) and leaf dry matter content (indicative of investment in structural strength) showed no significant trends across the elevation range. These results give insight into the phenotypic plasticity of $E$. pauciflora, and provide information on how the ecosystem may respond to climate change in the future.

\section{Introduction}

All around the world, plant species exhibit phenotypic variation across their ranges, because varying environmental conditions cause either the formation of hybrid zones through natural selection or variation within a species (Holman et al. 2011). Intraspecific variation may be due to phenotypic plasticity, namely the ability of a certain genotype to express a variety of phenotypes in response to environmental conditions (Andrew et al. 2010). 
This phenotypic change due to the environment leads to measurable correlations between the phenotype of plants and their microhabitat. Understanding these correlations enables more accurate predictions of how ecosystems will respond to climate change, providing information on the resilience of an ecosystem. This allows different ecosystems to be assessed for their vulnerability to climate change.

Alpine regions are seen to undergo a more rapid response to climate change than is seen in other areas, making them a priority in conservation efforts (Jurasinski and Kreyling 2007). These areas have variable and highly localised abiotic conditions, which are accompanied by high biodiversity and relatively sharp transitions between vegetation communities (Beniston 2003). Due to the complex nature of these ecosystems, change in the environment is thought to cause homogenisation of high altitude communities and loss of alpine biodiversity (Speed et al. 2012).

It is therefore important to explore the ways through which species are able to survive in variable conditions. In this study in the Australian Alps, Eucalyptus pauciflora, commonly known as snow gum, was chosen due to its abundance across a large altitudinal range and thereby across a range of abiotic conditions (O'Sullivan et al. 2013).

In order to explore the ways through which E. pauciflora is able to survive across different environments, the phenotypic variations in tree height, leaf size, leaf dry matter content (LDMC), leaf chlorophyll content and specific leaf area (SLA) of snow gum trees were measured across an altitudinal gradient. Due to increasing environmental disturbance, tree height and leaf size are usually seen to decrease with elevation (Wang et al. 2012; Royer et al. 2008). LDMC is related to the structural strength of the leaves, while SLA is related to potential photosynthetic and growth rates and therefore an inverse relationship with LDMC is often seen (Pescador et al. 2015). Chlorophyll content, the primary light-harvesting pigment in a plant's leaves, which varies in response to light and stress levels, is vitally important as photosynthetic potential and primary production is limited by the solar radiation absorbed by the plant (Turkis and Ozbucak 2010). In terms of chlorophyll content variability across an elevation gradient, conflicting results have previously been found (Filella and Peñuelas 1999; Covington 1975). 
The aim of this paper is to explore the phenotypic variation among E. pauciflora across an elevational gradient in the Australian Alps to gain insight into the species' ability to survive in a range of environmental conditions. Leaves are expected to be smaller, LDMC lower and SLA higher at lower elevations, while trees are expected to be shorter at higher elevations.

\section{Method}

\section{Field procedure}

Sampling was done at four different sites along an elevational gradient (shown in Table 1). At each site, eight leaves were taken from each of 10 different trees, and chlorophyll content (in SPAD units) of three of the leaves from each tree was measured three times with a chlorophyll meter and the measurements from each tree were then averaged. The height of each tree was calculated using angles measured with a clinometer after walking $10 \mathrm{~m}$ from the base of the tree. The global positioning system (GPS) coordinates of each tree were also recorded.

Table 1: Elevations of the four different sample sites

\begin{tabular}{|l|l|l|}
\hline Site & Name & Elevation $(\mathrm{m})$ \\
\hline 1 & Waste Point & 920 \\
\hline 2 & Kosciuszko Learning Centre & 1,200 \\
\hline 3 & Rainbow Lake & 1,600 \\
\hline 4 & Charlotte Pass & 1,860 \\
\hline
\end{tabular}

Source: Authors' data.

\section{Laboratory procedure}

In the laboratory, five leaves from each tree were scanned using ImageJ to give an average leaf area for each site, with the three remaining leaves being set aside for later genetic testing. The scanned leaves were then saturated with water by soaking for a minimum of 10 minutes, at which point they were measured using a fine scale balance to determine saturated leaf mass. The leaves were then placed in an oven overnight at $70-80^{\circ} \mathrm{C}$, after 
which they were again weighed to determine oven-dry leaf mass. These measurements were then used to calculate SLA and LDMC using the equations shown below.

$$
\begin{aligned}
S L A & =\frac{\text { Leaf Area }\left(\mathrm{mm}^{2}\right)}{\text { Leaf Dry Mass }(\mathrm{g})} \\
L D M C & =\frac{\text { Leaf Dry Mass }(\mathrm{g})}{\text { Saturated Dry Mass }(\mathrm{g})}
\end{aligned}
$$

\section{Results}

Trees at site 1 were significantly taller than those at Sites 2 and $3(P<0.05$, Figure 1a), and while trees at site 1 appeared to be taller than those at site 4 , this difference was not significant $(P>0.05$, Figure 1a), due to the large variance seen at site 4 . Sites 2,3 and 4 were also not significantly different from each other $(P>0.05$, Figure 1a).

In terms of leaf size, leaves were seen to decrease in size as elevation increased. Leaf area decreased as elevation increased, though the difference between the leaf size at sites 1 and 2 was not statistically significant $(P>0.05$, Figure $1 \mathrm{~b})$. Despite this, the decrease in leaf size from sites 2 to 3 and from sites 3 to 4 was seen to be statistically significant $(P<0.05$, Figure 1b). Leaf mass also appeared to decrease as elevation increased, and again the decrease in mass from sites 1 to 2 was not statistically significant $(P>0.05$, Figure 1c). The decrease in mass from sites 2 to 3 and from 3 to 4 though was statistically significant $(P<0.05$, Figure $1 \mathrm{c})$. No significant trend was seen in regards to SLA $(P>0.05$, Figure 1f).

In the analysis of leaf composition, no significant trend was seen in LDMC across the elevation gradient $(P>0.05$, Figure $1 \mathrm{~d})$, but a trend was seen in chlorophyll content. Sites 1 and 2 were seen to have significantly lower chlorophyll content than sites 3 and $4(P<0.05$, Figure $1 \mathrm{e})$. However, the difference in chlorophyll content between sites 1 and 2 and between sites 3 and 4 was not statistically significant $(P>0.05$, Figure $1 \mathrm{e})$. There were no significant differences in SLA between sites (Figure 1f). 
a)

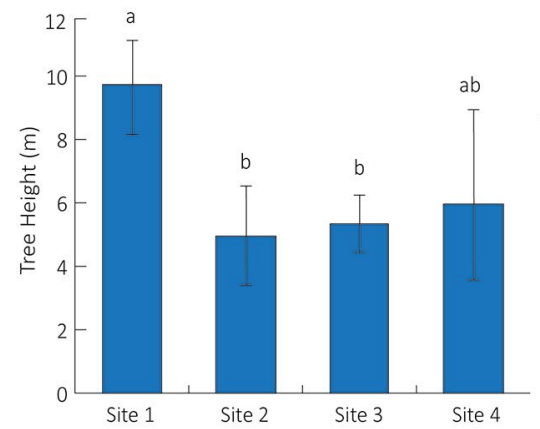

c)

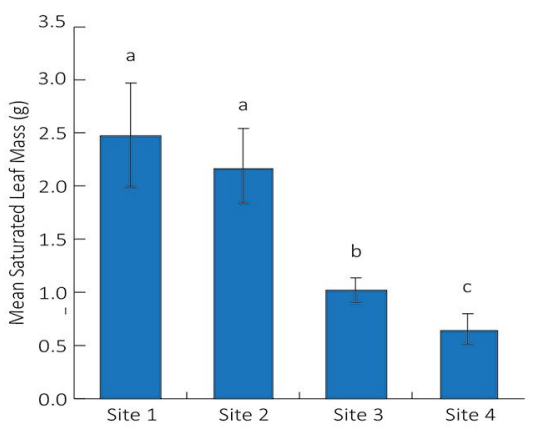

e)

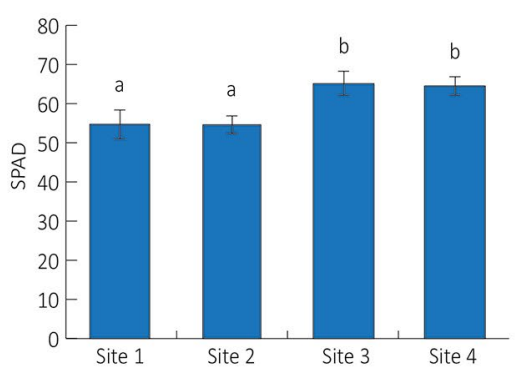

b)

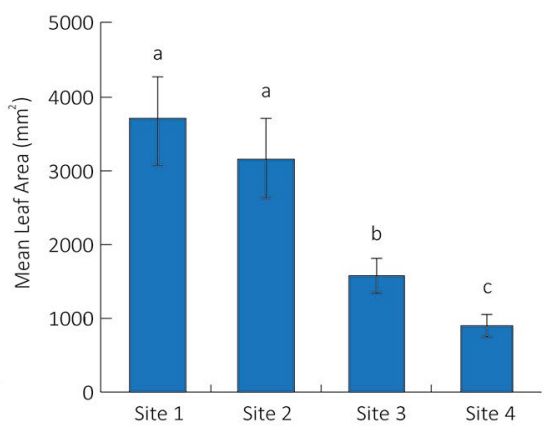

d)

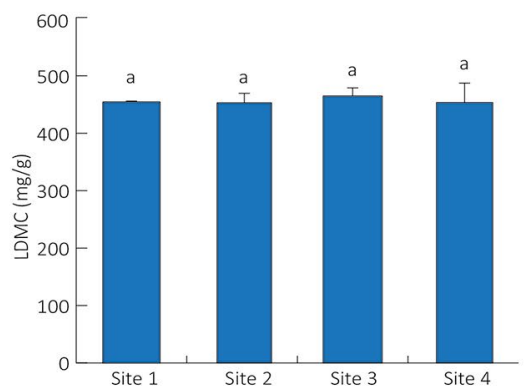

f)

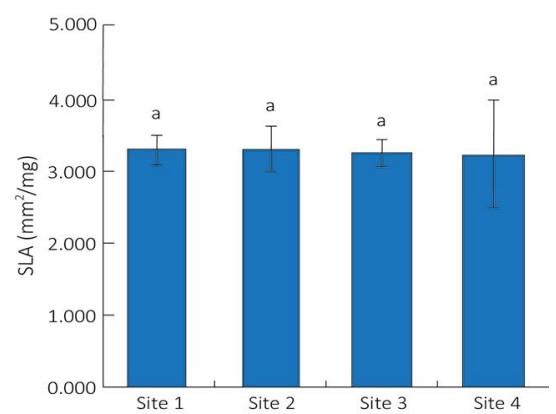

Figure 1: Column graphs showing variation in a) tree height, b) mean leaf area, c) mean saturated leaf mass, d) leaf dry matter content, e) chlorophyll content and f) specific leaf area across an elevational gradient. Site 1 was Waste Point (920 m), site 2 was Kosciuszko Learning Centre $(1,200 \mathrm{~m})$, site 3 was Rainbow Lake $(1,600 \mathrm{~m})$ and site 4 was Charlotte Pass $(1,860 \mathrm{~m})$.

Note: Error bars represent 95 per cent confidence intervals.

Source: Authors' data. 


\section{Discussion}

In order to better understand how E. pauciflora is able to survive in a range of environmental conditions, a number of plant traits were measured across an elevational gradient in the Australian Alps. As seen in Figure 1a, site 1 (the lowest elevation) had taller trees than higher elevation sites. At sites 2 and 3, sampling was limited to small trees due the fact that the leaves of tall trees (which were more abundant) were inaccessible given the equipment available. If this sampling issue could be resolved, it is expected that, given both the hypothesis and results seen previously in the literature (Wang et al. 2012), observations would show tree height decreasing as elevation increases,

In terms of leaf size, when analysed in regards to both leaf area and leaf mass (Figures 1b and 1c), a downward trend is apparent as elevation increased. This is consistent with our predictions and results seen in previous research (Royer et al. 2008).

The results for LDMC and SLA (Figures 1d and 1f) showed no significant trend across the elevational gradient. While these results do not support the hypothesis, previous studies have revealed varying trends in SLA and LDMC across elevational gradients (Zhong et al. 2014). The reason for this deviance from the hypothesis could be due to the genotype of $E$. pauciflora not allowing for significant phenotypic plasticity in regards to SLA or LDMC.

Chlorophyll content was also seen to change with elevation, with the leaves from sites 3 and 4 having significantly higher chlorophyll content than those from sites 1 and 2 . In previous studies, a variety of trends have been seen (Filella and Peñuelas 1999; Covington 1975). A possible explanation for the trend seen in this study is that, in order to maintain carbon uptake as leaf size decreases with elevation, an increased chlorophyll content is needed to increase the amount the overall solar radiation absorbed. Another explanation could be that chlorophyll content varies seasonally and in growth periods (Turkis and Ozbucak 2010), such that the observed difference in chlorophyll content may simply be due to differences in growth periods brought on by the varying microclimates at each site.

There is much room for further research into the phenotypic plasticity of E. pauciflora, especially in regards to LDMC, SLA and chlorophyll content. In the case of chlorophyll content, measuring it at various periods 
throughout the year in order to isolate the variable of growth periods may help to increase the accuracy of the results achieved. For SLA and LDMC, analysis of other species within the range may help to determine whether the absence of a clear trend in E. pauciflora was due to its genotype or simply the environment in which it is found. Research on additional plant traits could also be done. Additionally, in the analysis of the results achieved for chlorophyll content, leaf area and leaf mass, a clear break was seen between the observations of site 2 and 3 (see Figures 1b, 1c, 1e). Further sampling between sites 2 and 3 may determine whether there is a particular elevation at which traits change, or whether the significant difference in observations was due to the large distance between sites (both elevation and horizontal distance). For some measurements, namely tree height, LDMC and SLA, site 4 exhibited large variation. This may be due to there being more varied microhabitats present at such a high and exposed elevation. Future studies could also investigate the difference in microhabitats in these types of environments. Finally, the leaves set aside for later genetic testing could also provide definitive evidence as to whether the variation in phenotype is due to phenotypic plasticity or genetic variation.

The results achieved in this paper provide insight into the ability of E. pauciflora to thrive across such a range of environments. By learning which adaptions enable it to perform better in certain environments, we can better predict how the distribution and characteristics of the snow gum population will change as the climate warms. Through further research, both into E. pauciflora and other species, a better understanding can be gained of how the alpine ecosystem as a whole will respond to climate change.

\section{Acknowledgements}

We thank The Australian National University Research School of Biology for providing the materials needed to perform the experiment and Megan Supple for supervising the study. 


\section{References}

Andrew R, Wallis I, Harwood C, Foley W (2010) Genetic and environmental contributions to variation and population divergence in a broad-spectrum foliar defence of Eucalyptus tricarpa. Annals of Botany 105: 707-17. doi.org/10.1093/aob/mcq034

Beniston M (2003) Climatic change in mountain regions: A review of possible impacts. Climatic Change 59: 5-31. doi.org/10.1007/97894-015-1252-7_2

Covington W (1975) Altitudinal variation of chlorophyll concentration and reflectance of the bark of Populus tremuloides. Ecology 56: 715-20. doi.org/10.2307/1935507

Filella I, Peñuelas J (1999) Altitudinal differences in UV absorbance, UV reflectance and related morphological traits of Quercus ilex and Rhododendron ferrugineum in the Mediterranean region. Plant Ecology 145(1): 157-65. doi.org/10.1023/A:1009826803540

Holman J, Hughes J, Fensham R (2011) Origins of a morphological cline between Eucalyptus melanophloia and Eucalyptus whitei. Australian Journal of Botany 59: 244-52. doi.org/10.1071/BT10209

Jurasinski G, Kreyling J (2007) Upward shift of alpine plants increases floristic similarity of mountain summits. Journal of Vegetation Science 18: 711-18. doi.org/10.1658/1100-9233(2007)18[711:USO API 2.0.CO;2

O'Sullivan OS, Weerasinghe K, Evans J, Egerton J, Tjoelker M, Atkin O (2013) High-resolution temperature responses of leaf respiration in snow gum (Eucalyptus pauciflora) reveal high-temperature limits to respiratory function. Plant, Cell and Environment 36: 1268-84. doi. org/10.1111/pce. 12057

Pescador D, De Bello F, Valladares F, Escudero A (2015) Plant trait variation along an altitudinal gradient in Mediterranean high mountain grasslands: Controlling the species turnover effect. PLoS ONE 10: e0118876. doi.org/10.1371/journal.pone.0118876 
Royer D, McElwain J, Adams J, Wilf P (2008) Sensitivity of leaf size and shape to climate within Acer rubrum and Quercus kelloggii. New Phytologist 179: 808-17. doi.org/10.1111/j.1469-8137.2008.02496.x

Speed J, Austrheim G, Hester A, Mysterud A (2012) Elevational advance of alpine plant communities is buffered by herbivory. Journal of Vegetation Science 23: 617-25. doi.org/10.1111/j.16541103.2012.01391.x

Turkis S, Ozbucak T (2010) Foliar resorption and chlorophyll content in leaves of Cistus creticus L. (Cistaceae) along an elevational gradient in Turkey. Acta Botanica Croatica 69: 275-90.

Wang Y, Čufar K, Eckstein D, Liang E (2012) Variation of maximum tree height and annual shoot growth of smith fir at various elevations in the Sygera Mountains, southeastern Tibetan Plateau. PLoS ONE 7: e31725. doi.org/10.1371/journal.pone.0031725

Zhong M, Wang J, Liu K, Wu R, Liu Y, Wei Z, Pan D, Shao X (2014) Leaf morphology shift of three dominant species along altitudinal gradient in an alpine meadow of the Qinghai-Tibetan Plateau. Polish Journal of Ecology 62: 639-48. doi.org/10.3161/104.062.0409 
This text is taken from Researching functional ecology in Kosciuszko

National Park, edited by Hannah Zurcher, Chia Ming-Dao, Michael Whitehead and Adrienne Nicotra, published 2017 by ANU eView, The Australian National University, Canberra, Australia. 